\title{
Status of the two-dimensional, multi-wire proportional chamber detector for the China Spallation Neutron Source
}

\author{
Zhiwen Wen \\ School of Nuclear Science and Technology, Lanzhou University \\ Lanzhou 730000, China \\ wenzhw@ihep.ac.cn \\ Huirong Qi \\ State Key Laboratory of Particle Detection and Electronics \\ Institute of High Energy Physics, Chinese Academy of Sciences \\ Beijing 100049, China \\ qihr@ihep.ac.cn
}

Published 10 July 2018

\begin{abstract}
The re-designed two-dimensional, multi-wire proportional chamber (MWPC) detector based on the ${ }^{3} \mathrm{He}$ operation gas has been developed for the multifunctional reflection spectrum detection requirements in China Spallation Neutron Source (CSNS), which is under construction in Guangdong province, China. This efficient thermal neutron detector with large area (200 $\mathrm{mm} \times 200 \mathrm{~mm}$ active area), two-dimensional position sensitive $(<2 \mathrm{~mm}$ of position resolution), high detection efficiency ( $>65 \%$ in the wavelength of $1.8 \AA$ ) and $\operatorname{good} \mathrm{n} / \gamma$ discrimination would meet some requirements in CSNS. The neutron detector consists of a MWPC detector and a high-pressure gas vessel. The wire readout structures of the detector and the gas purity device have been optimized based on previous design and testing. The re-designed MWPC detector with an absorber thickness of $10 \mathrm{~mm}$ and $8.5 \mathrm{~atm}$ operating gas mixture of ${ }^{3} \mathrm{He}$ and $\mathrm{C}_{3} \mathrm{H}_{8}$ was constructed. Using the non-return valve manufactured by Swagelok, the gas purity device was developed to clean the water and remove gas impurities. The effective cycle time can be up to $50 \mathrm{~min}$ per sequence. The performance of the position resolution and the two-dimensional imaging accuracy by the traditional center of gravity readout method was studied with an X-ray radiation source and the neutron source. At the end of this year, the detector will be mounted at CSNS and studied using the neutron source.
\end{abstract}

Keywords: Multi-wire proportional chamber; Position resolution.

PACS numbers: 29.40.Cs, 29.40.Gx

\section{Introduction of CSNS and MR}

The China Spallation Neutron Source (CSNS), which is under construction in Guangdong province, China, will be the first spallation neutron source in the developing countries.

This is an Open Access article published by World Scientific Publishing Company. It is distributed under the terms of the Creative Commons Attribution 4.0 (CC-BY) License. Further distribution of this work is permitted, provided the original work is properly cited. 
The design beam power of the CSNS is from $120 \mathrm{~kW}$ to $500 \mathrm{~kW}$ for the first stage and the next update stage. At the repetition rate of $25 \mathrm{~Hz}$, the accelerators can deliver a beam power of $120 \mathrm{~kW}$ at Phase I of the CSNS stages. The neutron scattering detector techniques have been playing a progressively more important role in biomedical physics, biophysics, condensed matter physics, and within chemical research fields as compensation for synchrotron radiation X-rays.

During Phase I, there are three spectrometers and detectors for neutron detection. The first neutron instrument is the high-intensity powder diffractometer. The powder diffractometer is designed for general purpose powder diffraction with good resolution of $\sim 0.2 \%$ or less value. Its general purpose is to determine crystallographic and magnetic structures. The second instrument is a small-angle neutron scattering mechanism. It is designed to probe material structure and correlations on the scale of nanometers. The third and final instrument is a multipurpose reflectometer (MR) used to obtain the interface structure for films. The MR diffractometer - as one of the three spectrometers to be built in the first phase project-requires a thermal neutron detector in the wavelength range from $0.4 \AA$ to about $8 \AA$. Such a detector should have an active area of $200 \mathrm{~mm} \times 200 \mathrm{~mm}$, and a position resolution of less than $2 \mathrm{~mm}$. The position sensitive neutron detector originates from the multi-wire proportional chamber (MWPC) and is based on the ${ }^{3} \mathrm{He}$ convertor. ${ }^{1}$ The detector has high detection efficiency and good $\mathrm{n} / \gamma$ discrimination for the neutron source.

In this paper, development of the re-designed two-dimensional MWPC detector based on ${ }^{3} \mathrm{He}$ operation gas is discussed, and some preliminary, obtained results are presented. The results demonstrate that the detector meets the physics requirements of MR detection for CSNS.

\section{MWPC Detector System}

To meet the physics requirements of CSNS, the thermal neutron, a neutral particle, is usually detected indirectly through the nuclear interaction with other nuclides such as ${ }^{3} \mathrm{He},{ }^{6} \mathrm{Li}$, or ${ }^{10} \mathrm{~B} .{ }^{2}$ The ${ }^{3} \mathrm{He}$ gas is a very good choice for the MWPC detector. When ${ }^{3} \mathrm{He}$ gas reaches $6 \mathrm{~atm}$, the detection efficiency of the neutron will reach up to $70 \%$ at a wavelength of $2 \AA$. In order to improve the position resolution, the secondary particles need to be limited into a small range. To accomplish this, another stopping gas is needed, such as $\mathrm{C}_{3} \mathrm{H}_{8}, \mathrm{CF}_{4}$, or $\mathrm{iC}_{4} \mathrm{H}_{10}{ }^{3,4}$

In the test, the stopping gas should be considered as an additional operation gas because it has two roles to perform. First, it must reduce the path length of the electrons for a good position resolution and minimize the wall effects. Second, in an environment of high photon background, it must have a low sensitivity to gamma rays and X-rays. $\mathrm{iC}_{4} \mathrm{H}_{10}$ liquefies at about $2 \mathrm{~atm}$ and is a potential environmental pollutant of $\mathrm{CF}_{4}$. $^{5,6}$ Therefore, we elected to use $\mathrm{C}_{3} \mathrm{H}_{8}$ (2.5 atm) as the stopping gas.

The neutron detector consists of a MWPC and a high-pressure gas chamber. The MWPC consists of four planes and one absorption region as shown below in Fig. 1. From top to bottom, these are the cathode plane, the absorption region of $14 \mathrm{~mm}$ filled the 
operation gas, the $\mathrm{X}$-axis readout plane of the wire with $2.0 \mathrm{~mm}$ pitch, the anode plane of the wire with $4.0 \mathrm{~mm}$ pitch as shown in Fig. 1, and the Y-axis readout plane of the goldplated strip in the printed circuit board (PCB). The wire tensions were measured by the electrostatic resonance method and had a good consistency of less than $5 \%$.

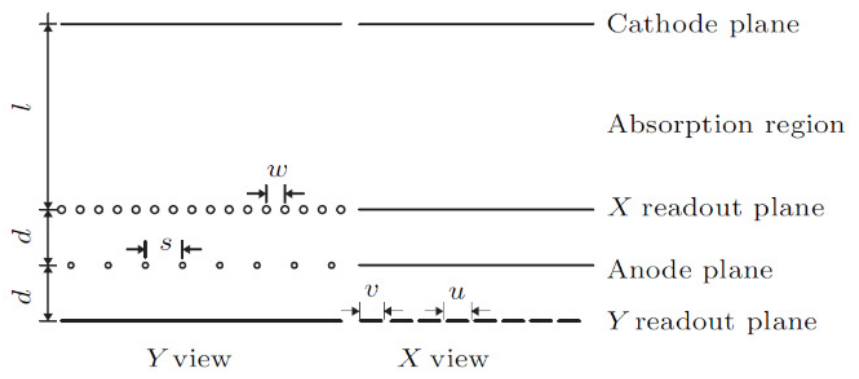

Fig. 1. Diagram of the detector structure.

To optimize the detector performance, it is important to focus on gas purity. This is achieved by cycling the working gas through a gas purity device for 30-50 min in order to clear the oxygen and water from the gas.

\section{Observed Detector Results}

For the wire detector optimization, four sets of wire detectors were developed and assembled by the same process in our lab. All of the wire detectors were tested in the same gaseous chamber with gas flow of $30 \mathrm{~mL} / \mathrm{min}$ using the $\mathrm{X}$-ray radioactive source of ${ }^{55} \mathrm{Fe}$. The operation voltage of the anode wire and the plateau curve are shown in Table 1. Set \#3 was selected for the test, and the other sets were used as backup devices for the detector.

Table 1. Four sets of the wire detectors.

\begin{tabular}{ccccc}
\hline Items & Set \#1 & Set \#2 & Set \#3 & Set \#4 \\
\hline Operation voltage of the anode wire (V) & 2150 & 2150 & 2150 & 2150 \\
Plateau curve (V) & $1700-2030$ & $1700-2030$ & $1650-1990$ & $1650-1910$ \\
Voltage range of the curve (V) & 360 & 330 & 340 & 260 \\
Linearity of the Gain & $16.5 \%$ & $13.5 \%$ & $7.76 \%$ & $7.51 \%$ \\
\hline
\end{tabular}

\subsection{Detector and operating gas}

The performance of the position resolution and the two-dimensional imaging accuracy by the traditional center of gravity readout method was studied with an X-ray radiation source. From Table 1 above, the wire detector of Set \#3 was assembled in the highpressure vessel. Aluminum alloy T7500 was used considering its high-pressure load and least neutron reflection. Figure 2 shows the assembled detector filled with the operation 
gas at $8.65 \mathrm{~atm}$. All the electrodes were manufactured on the PCB except for the cathode, which was made on the aluminum window with a thickness of $9.0 \mathrm{~mm}$ at the top to minimize the thickness of material in the entry window. After all the planes of the detector were assembled, the MWPC was placed into a container made from the stainless-steel chamber. The operation gas filled in $2 \mathrm{~h}$, and the fill working gas flow was kept at a low level $(\sim 0.01 \mathrm{~atm} / \mathrm{min})$.
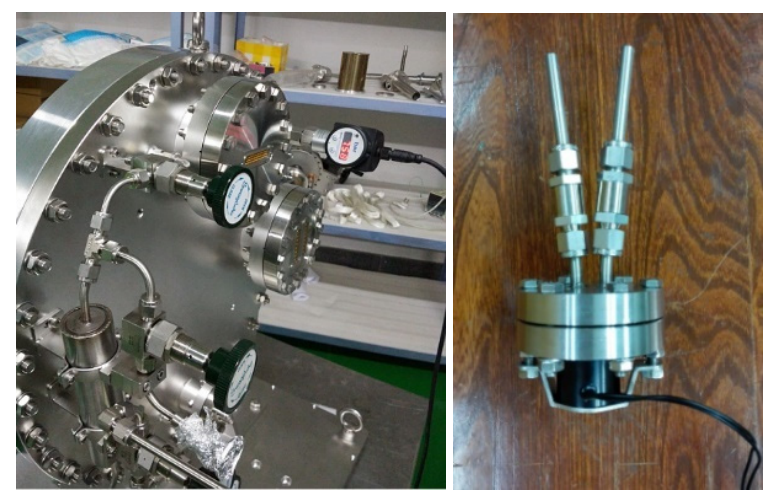

Fig. 2. The redesigned $2 \mathrm{D}$ detector (left) and the purity device (right).

The purity device was integrated into the detector system. The material of the molecular sieves inside the device cleared and purified the oxygen and water to the parts per million (ppm) level in the working gas. Figure 3 shows the variation of the operating gas pressure inside the detector as a function of temperature and time: the black line is the pressure variation with time, and the red line is the pressure variation with room temperature. The pressure testing was recorded for approximately 32 days in the lab, and three readings were recorded each day. The new designed detector vessel performed well for this high-working pressure.

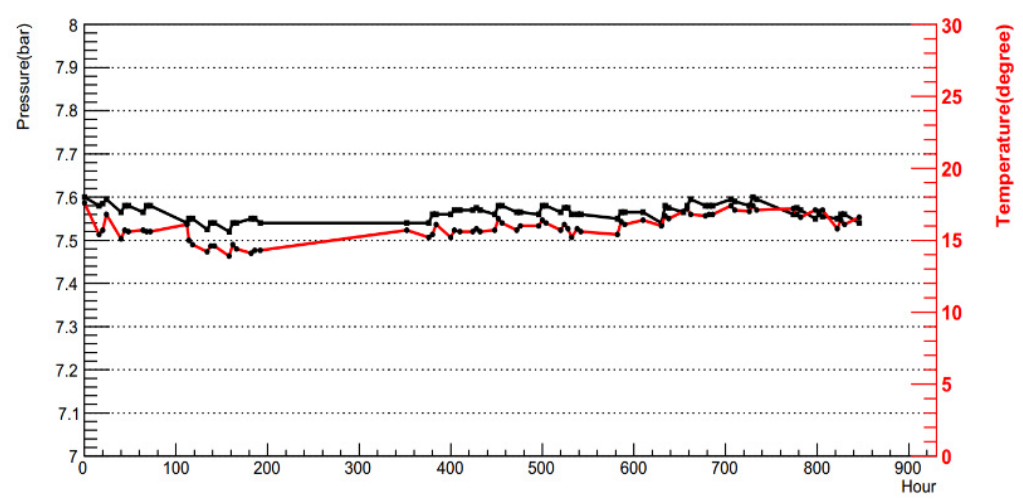

Fig. 3. Gas pressure as a function of time and temperature. 


\subsection{Gas purity and heating process}

To minimize the influence of water in the wire PCB and chamber, the heating process should be done before the chamber is assembled in the lab. When the detector is finished, gas impurities can be removed with the integration of the gas purity device.

The PCB with wires was heated in the high temperature vessel, and the temperature reached between $80^{\circ} \mathrm{C}$ to $150^{\circ} \mathrm{C}$. The results showed that the wire will lose tension if the temperature is greater than $120^{\circ} \mathrm{C}$. In the heating process, the temperature was selected at $105^{\circ} \mathrm{C}$ to clear water in the PCB and retain wire tension. Figure 4 shows that the count rate changes with or without the heating process for the wire PCBs. It obviously indicates that the wire PCBs and the support boards in the chamber should be used in the heating process to improve detector performance.
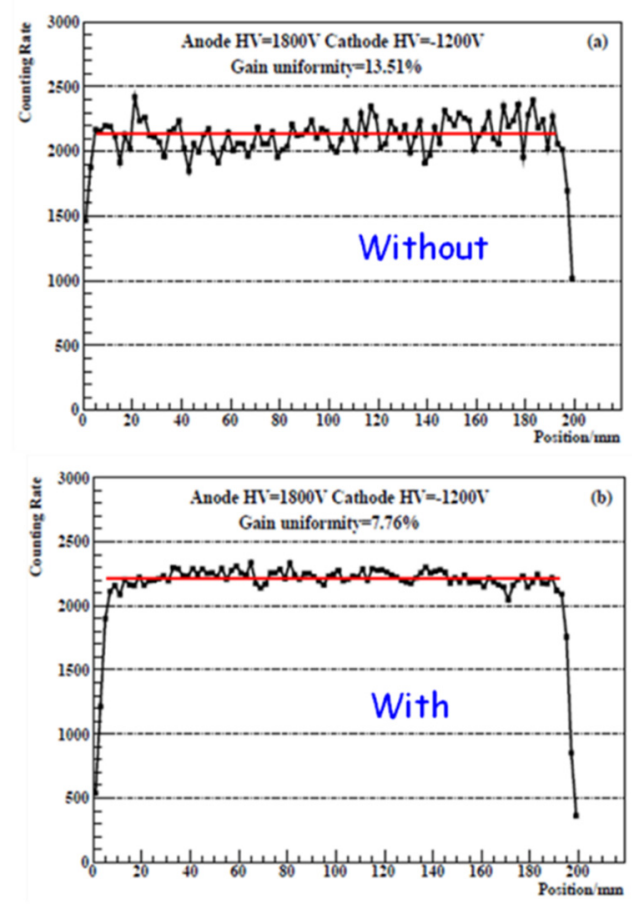

Fig. 4. Count rate with/without the wire heating process.

\subsection{Imaging and position resolution}

The two-dimensional imaging capability and the position resolution were very important parameters for the detector. Some preliminary results have been obtained by the same geometry prototype at the $\mathrm{Cf}$ neutron radioactive source in the lab.

When a neutron particle was injected into the detector by passing through a cadmium collimator with a diameter of $2 \mathrm{~mm}$ and a depth of $2 \mathrm{~mm}$, the response of the detector was measured and shown below in Fig. 5. A reconstructed two-dimensional image could 
be obtained from the results. The position resolution parallel to the direction of the anode wires was tested by the Cf source collimated by a slit of $0.8 \mathrm{~mm}$ wide and $10.0 \mathrm{~mm}$ thick. The position spectrum was fitted using the function of the convolution of the Gaussians function and the result is again shown below in Fig. 5. A position resolution of $1.23 \mathrm{~mm}$ $\pm 0.02 \mathrm{~mm}$ FWHM was obtained. If the collimator is placed by a slit $2.0 \mathrm{~mm}$ wide and $10.0 \mathrm{~mm}$ thick, then the best position resolution that could be reached was $1.22 \mathrm{~mm} \pm$ $0.02 \mathrm{~mm}$ FWHM.
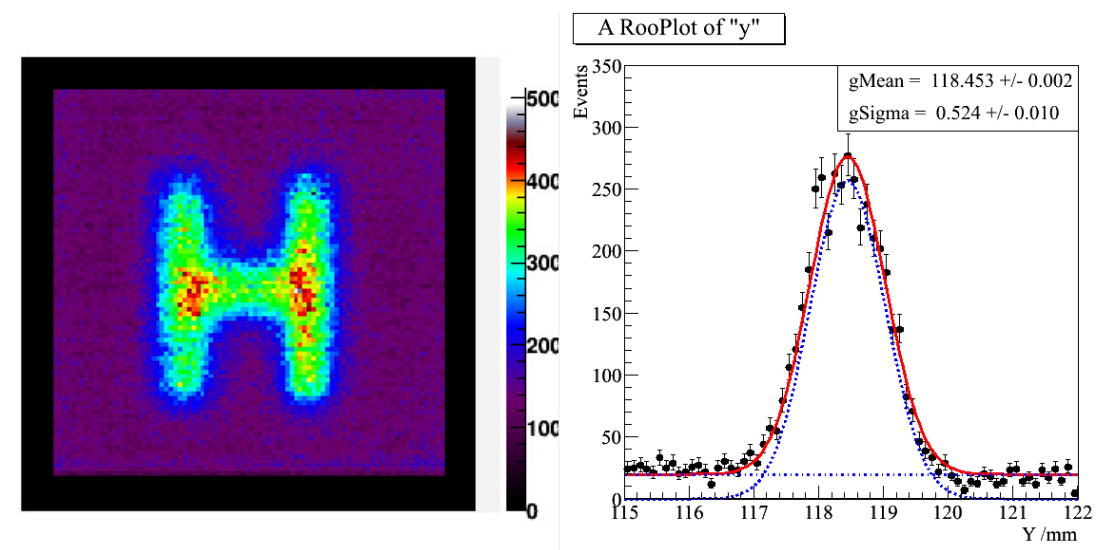

Fig. 5. 2D imaging (left) and the position resolution (right).

\section{Conclusion}

The two-dimensional MWPC detector based on the ${ }^{3} \mathrm{He}$ operation gas has been constructed and tested to verify the operational specifications for the multifunctional reflection in CSNS. The detector consisted of a MWPC and a high-pressure vessel.

Its performance has been tested and preliminary results have been obtained. The results show that the detector could be fitted to the physics requirements. At the end of this year, the detector system will be assembled in the MR site of the CSNS project.

\section{Acknowledgments}

This work was supported by the National Natural Science Foundation of China (Grant No. 11675197 and Grant No. 11775242).

\section{References}

1. J. Orban, L. Cser, L. Rosta, Gy. Török, and A. Nagy, Nucl. Instr. Meth. A 632, 124 (2011).

2. X. L. Li, Y. L. Zhang, H. Qian, Z. Z. Xu, and X. L. Wang, Chin. Phys. C 36, 519 (2008).

3. R. A. Boie, J. Fischer, Y. Inagaki et al., Nucl. Instr. Meth. 200, 533 (1982).

4. M. K. Kopp, K. H. Valentine, L. G. Christophorou, Nucl. Instr. Meth. 201, 395 (1982).

5. V. Radeka, R. A. Boie, Nucl. Instr. Meth. 178, 543 (1980).

6. P. Fonte, Nucl. Instr. Meth. A 348, 338 (1994). 